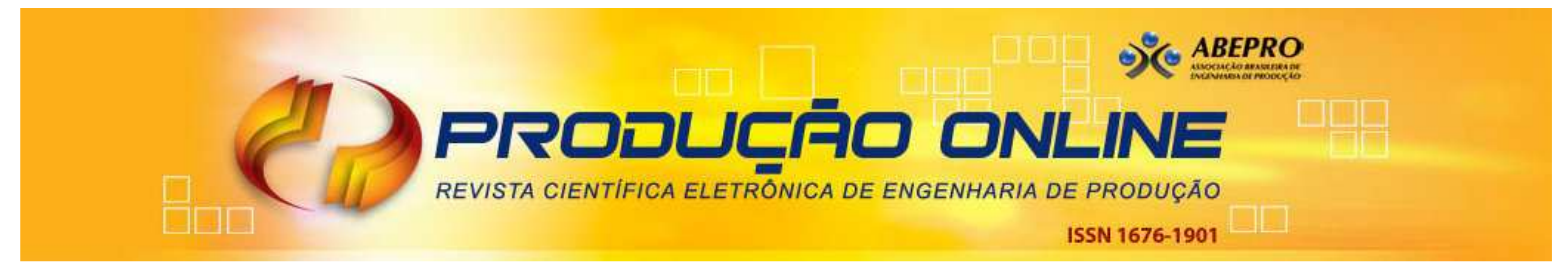

\title{
UTILIZAÇÃO DA TI PARA TRANSFERÊNCIA DE CONHECIMENTOS ENTRE EQUIPES DE DESENVOLVIMENTO DE PRODUTO: COMPARAÇÃO ENTRE EQUIPES VIRTUAIS E CO-LOCALIZADAS
}

\section{USING IT FOR KNOWLEDGE TRANSFER BETWEEN PRODUCT DEVELOPMENT TEAMS: A COMPARISON BETWEEN VIRTUAL AND COLLOCATED TEAMS}

\author{
Alejandro Germán Frank*E-mail: agerfrank@producao.ufrgs.br \\ José Luis Duarte Ribeiro* E-mail: ribeiro@producao.ufrgs.br \\ *Universidade Federal do Rio Grande do Sul (UFRGS), Porto Alegre, RS
}

\begin{abstract}
Resumo: A utilização das tecnologias da informação (TI) para a transferência de conhecimentos (TC) entre equipes de desenvolvimento de produto depende da estratégia de trabalho adotada, podendo ser trabalho em equipes virtuais ou co-localizadas. Assim sendo, este artigo tem por objetivo analisar as características da TI utilizada para a TC nestes dois tipos de estratégias, comparando as diferenças entre as mesmas. Para tanto, foi realizado um estudo de caso em duas empresas multinacionais do setor de máquinas agrícolas, cada uma destas com um tipo diferente de estratégia de trabalho de equipes de projeto. Observou-se que a empresa com equipes co-localizadas utiliza TI principalmente para a retenção de conhecimento em fontes explícitas, uma vez que a TC acontece principalmente por canais informais que levam ao risco de perda do conhecimento. Por outro lado, a empresa com equipes virtuais investe em tipos de TI que ajudem a superar as distâncias físicas e culturais entre os participantes de diferentes países. Os resultados também apresentam uma análise comparativa das características da $\mathrm{TI}$ em cada estratégia, utilizando-se para isto uma abordagem sociotécnica que considera quatro áreas de análises na utilização da TI: Pessoas, Tecnologias, Organização do Trabalho e Meio Ambiente.
\end{abstract}

Palavras-chave: Tecnologias da Informação. Transferência de conhecimentos. Equipes virtuais. Equipes co-localizadas. Desenvolvimento de produtos.

\begin{abstract}
The mean in which Information Technology (IT) is used for knowledge transfer (KT) between product development project teams depends on the adopted strategy for team working (i.e. virtual teams or collocated teams). This paper aims to analyze the characteristics of the IT used for KT in these two work team strategies, comparing differences between them. In order to achieve this aim, a case study was carried out in two multinational companies from the agriculture machinery industry, each one of them with a different team work strategy. Results show that the company with collocated teams uses IT mainly for knowledge retention in explicit sources. This is because in such case KT occurs by informal channels that lead to knowledge losing risks. On the other hand, company with virtual team strategy uses types of IT to help overcome cultural and physical distances among members. This paper also presents a comparative analysis between both team work strategies by means of a framework based on a socio-technical approach. This approach subdivides IT use in four categories of analysis: (i) Technologies; (ii) People involved; (iii) Work design and (iv) External environment influence.
\end{abstract}

Keywords: Information technology. Knowledge transfer. Virtual teams. Collocated teams. Product development. 


\section{INTRODUÇÃO}

A tendência do aumento dos produtos a serem desenvolvidos nas empresas faz com que sejam necessários cada vez mais recursos para execução dos projetos de produto, além de crescer também a complexidade do gerenciamento de projetos (MARSH e STOCK, 2006; CORSO e PAVESI, 2000; NOBEOKA e CUSUMANO, 1997).

Considerando esta realidade, não basta ser eficiente em cada projeto individual, mas é necessário assegurar o desempenho de todo o conjunto de projetos que utiliza a mesma fonte de recursos (CUSUMANO e NOBEOKA, 1998; CORSO et al., 1999).

Dentre os recursos que devem ser gerenciados considera-se o conhecimento das equipes o mais importante e estratégico, uma vez que estabelece a base para a vantagem competitiva da empresa. Isto é especialmente importante nas empresas que possuem um processo de desenvolvimento de produtos intensivo em criação de novos conhecimentos dentro de cada equipe de produto (ZHENGFENG, 2007). O conhecimento criado em cada equipe de desenvolvimento de produto precisa ser utilizado posteriormente em projetos sucessivos ou em outras equipes que trabalham em paralelo para obter o máximo aproveitamento deste recurso. $\mathrm{O}$ aproveitamento do conhecimento entre diferentes equipes é muitas vezes denominado como transferência de conhecimentos (TC) entre projetos.

Embora seja necessária a TC entre as equipes, em muitos casos a mesma é difícil de acontecer naturalmente. Isto é devido ao fato de que famílias de produtos diferentes não têm uma vinculação direta entre si e as equipes se concentram apenas nos seus próprios objetivos de projeto (BARTEZZAGHI et al., 1997; AOSHIMA, 2002).

Além disso, a forma de integração entre as equipes depende da organização, seja em equipes co-localizadas (compartilhamento do mesmo ambiente de trabalho) ou em equipes virtuais (equipes geograficamente distanciadas). Song et al. (2007) destacam que ambas as formas de organização das equipes requerem do suporte da Tecnologia da Informação (TI) para que a TC seja bem sucedida. Neste sentido, vários estudos têm se concentrado nas formas de contribuição da TI para integração entre as equipes (por ex.: Lynn et al., 2000; Sarker et al., 2005; Alavi e Leidner, 2001, entre outros). Contudo, esses trabalhos não têm focalizado suas análises na questão de Revista Produção Online, Florianópolis, SC, v.12, n. 4, p. 1106-1130, out./dez. 2012. 
como a TI é utilizada em cada estratégia particular de trabalho, seja em equipes virtuais ou co-localizadas.

Assim sendo, o objetivo deste artigo é identificar as principais características dos tipos de configurações de TI utilizados para TC entre projetos de produtos estruturados em equipes virtuais e equipes co-localizadas, e comparar a forma de utilização de $\mathrm{Tl}$ em cada uma dessas estratégias. Para tanto, o trabalho apresenta um estudo de caso em duas empresas multinacionais do setor de máquinas agrícolas que operam com diferentes estratégias de gestão de projetos de produto. A comparação dos casos é realizada por meio de uma análise baseada na abordagem sociotécnica de Hendrick e Kleiner (2003) que propõem quatro unidades de análises ou subsistemas: (i) Pessoas; (ii) Tecnologias; (iii) Organização do Trabalho e (iv) Ambiente externo. Por meio dessas unidades de análises é comparada a utilização da $\mathrm{TI}$ em ambos os casos e são destacadas as diferenças observadas.

\section{ESTRATÉGIAS DE TRABALHO DAS EQUIPES DE PROJETO DE PRODUTOS}

As atividades dos projetos de desenvolvimento de produtos podem ser realizadas por meio de duas estratégias principais de trabalho: equipes de projetos co-localizadas e equipes virtuais - ou por meio de uma abordagem mista combinando ambas (BARCZAK et al., 2008; SONG et al., 2007).

A estratégia de equipes co-localizadas consiste em equipes que compartilham o mesmo ambiente físico, inclusive quando os integrantes pertencem a diferentes áreas funcionais (BARCZAK et al., 2008). Esta é a forma mais tradicional de organizar as equipes de projetos para incentivar o trabalho colaborativo e a comunicação face-aface, visando especialmente a disseminação do conhecimento tácito (SONG et al., 2007; SCARBROUGH et al., 2004; NONAKA, 1994; LAKEMOND e BERGGREN, 2006).

Como neste tipo de estratégia prioriza-se a interação face-a-face entre os integrantes, a TI é utilizada apenas para o suporte, geralmente por meio do armazenamento de informações e registros dos projetos. Nestes casos a TI não atua como elemento-chave de funcionamento do trabalho colaborativo, mas como instrumento de suporte (TSENG, 2007).

Revista Produção Online, Florianópolis, SC, v.12, n. 4, p. 1106-1130, out./dez. 2012. 
Por outro lado, em muitas empresas as equipes começam a ser organizadas de uma maneira diferente à forma tradicional de equipes co-localizadas. Isto se deve à internacionalização das atividades de desenvolvimento de produtos (SONG et al., 2007). Neste caso, o trabalho é estruturado por meio de equipes geograficamente distanciadas que trabalham em conjunto de maneira virtual (SONG et al., 2007; MONTOYA et al., 2009). As equipes virtuais - ou também denominadas na literatura como equipes dispersas - consistem em um grupo de pessoas cujas atividades dos projetos transcendem as barreiras de tempo, espaço e da organização.

Estas equipes estão espalhadas por diferentes lugares trabalhando pelo mesmo objetivo e de maneira coordenada (MONTOYA et al., 2009; SMITH e BLANCK, 2002). Com esta estratégia se ganha na proximidade aos diferentes mercados e na flexibilidade dos projetos, pois não se precisa que o integrante esteja no local onde é desenvolvida a principal parte do projeto (SONG et al., 2007; SMITH e BLANCK,2002). Como contrapartida, a falta de interação face-a-face faz com que exista um risco de perda da capacidade de disseminação de conhecimentos entre as pessoas. Por esta razão, diferentes ferramentas baseadas em TI são utilizadas como elemento-chave que possibilita a criação de equipes virtuais bem sucedidas (MONTOYA et al., 2009).

\section{UTILIZAÇÃO DA TI PARA A TC EM EQUIPES VIRTUAIS E CO-LOCALIZADAS}

Um dos aspectos estudados na literatura sobre TC entre equipes de projeto é a influência e utilização da TI para este fim. No Quadro 10 são apresentados alguns exemplos de ferramentas de TI citadas como facilitadores da TC. A maior parte dessas ferramentas está associada à dimensão explícita do conhecimento. Todavia, no mesmo quadro aparecem também ferramentas de comunicação em tempo real. Estas estão vinculadas principalmente à dimensão tácita do conhecimento, pois nesses casos existe troca de experiências por meio da comunicação direta entre pessoas (SÖDERQUIST, 2006). 
Quadro 1 - Tecnologias da Informação para a TC entre projetos de produto

Ferramentas de TI para a TC

Autores

\begin{tabular}{ll}
\hline $\begin{array}{l}\text { Sistemas de armazenamento, compartilhamento ou } \\
\text { gerenciamento de arquivos/dados/ informações (Lotus Notes, } \\
\text { Repositórios, etc.) }\end{array}$ & $\begin{array}{l}\text { Lynn et al. (2000); Sarker et al. } \\
\text { (2005), Liu e Ke (2007). }\end{array}$ \\
\hline $\begin{array}{l}\text { Ferramentas de comunicação em tempo real (vídeo-conferência, } \\
\text { chats, conferência telefônica, etc.) }\end{array}$ & $\begin{array}{l}\text { Baskerville e Dulipovici (2006); } \\
\text { Sarker et al. (2005); Nonaka (1994) }\end{array}$ \\
\hline Intranets/extranet/portais de conhecimento & Baskerville e Dulipovici (2006) \\
\hline Trabalhos em redes & $\begin{array}{l}\text { Lynn et al. (2000); Sarker et al. } \\
\text { (2005) }\end{array}$ \\
\hline $\begin{array}{l}\text { Groupware e outras ferramentas de edição em grupo e acesso } \\
\text { geral }\end{array}$ & $\begin{array}{l}\text { Baskerville e Dulipovici (2006); } \\
\text { Prencipe e Tell (2001). }\end{array}$ \\
\hline Workflow & Baskerville e Dulipovici (2006) \\
\hline $\begin{array}{l}\text { Sistemas Baseados em Conhecimentos (Knowledge Based } \\
\text { Systems - KBS) }\end{array}$ & $\begin{array}{l}\text { Baskerville e Dulipovici (2006); Liu e } \\
\text { Ke (2007); Alavi e Leidner (2001). }\end{array}$ \\
\hline Ferramentas de divulgação eletrônica (electronic publishing, e- & $\begin{array}{l}\text { Baskerville e Dulipovici (2006); } \\
\text { mails, etc.) }\end{array}$ \\
\hline $\begin{array}{l}\text { Ferramentoya et al. (2009) } \\
\text { currículums de captura e gerenciamento de biografias e }\end{array}$ & Davenport e Völpel (2001). \\
\hline
\end{tabular}

Várias pesquisas têm discutido a capacidade dessas ferramentas de TI para integrar conhecimentos entre as equipes (por ex.: Cummings e Teng, 2003; Lin e Lee, 2006; Tseng, 2007; Zhengfeng, 2007 e Song et al., 2007). Algumas dessas pesquisas concluem que a TI é um meio efetivo para fornecer suporte e acesso aos conhecimentos das equipes. Essas ferramentas de TI ajudam a promover a integração inter-funcional e inter-organizacional mediante a facilitação da comunicação e colaboração entre diferentes equipes e o acesso a fontes explícitas de conhecimento (DAVENPORT e PRUSAK, 1998; CORSO et al., 2003; LYNN et al., 2000; CORSO e PAOLUCCI, 2001; CORSO et al., 2003; BOER et al., 2001; CHOI e LEE, 2003).

Corso e Paolucci (2001) e Choi e Lee (2003) destacam que, para o sucesso da integração de conhecimentos entre diferentes equipes, precisa-se da combinação destas ferramentas tecnológicas com interação face-a-face entre as pessoas. Os resultados de Song et al. (2005) constatam também isto, destacando uma forte contribuição tanto da TI como da interação face-a-face para a aplicação do conhecimento no desenvolvimento do produto. Além disso, em outra pesquisa, Song et al. (2007) estudaram o efeito da TI para equipes virtuais e co-localizadas sobre a TC. 
Esta pesquisa determina a necessidade de um balanceamento entre investimentos nos aspectos humanos e tecnológicos para o sucesso da TC, uma vez que nenhum deles isoladamente é a melhor estratégia a ser seguida.

Por outro lado, o estudo realizado por Barczak et al. (2008) analisa vários fatores que influenciam na utilização da Tl em projetos de desenvolvimento de produtos, dentre eles a co-localização das equipes. Os resultados mostraram que, quando se utiliza a estratégia de co-localização a importância atribuída à utilização de ferramentas de TI é baixa. Em contraste, Akgün et al. (2008) estudaram a utilização da TI dentro de equipes de desenvolvimento de produto independentemente da forma das equipes estarem localizadas. Os resultados desta pesquisa assinalam que a utilização da $\mathrm{Tl}$ durante o processo de desenvolvimento ajuda à inteligência das equipes de projeto. Dentre as ferramentas de TI estudadas por estes autores destacam-se: sistemas de e-mail, painéis de mensagens, notificações eletrônicas de comunicação, websites, etc. Todas estas ferramentas são também muito utilizadas nas equipes co-localizadas.

Outro estudo, de Montoya et al. (2009), analisou a utilização de TI em equipes virtuais. Estes autores assinalam diferentes padrões de comportamento das empresas em relação ao tipo de TI utilizadas e ressalta a contribuição de diferentes tipos de TI para o sucesso da integração entre equipes virtuais. Porém, estes resultados não foram comparados com o padrão de comportamento da TI nas equipes co-localizadas. Outras pesquisas que também abordam a questão de equipes virtuais e a importância da $\mathrm{TI}$ para as mesmas são as de Sarker et al. (2005), Joshi et al. (2007), Farshchian (2001), Ramesh e Tiwana (1999) e Tiwana e Ramesh (2001).

As pesquisas mencionadas anteriormente apresentam um denominador comum: todas elas destacam a necessidade de combinar fatores tecnológicos e práticas de socialização para a integração entre pessoas. Isto significa que essas pesquisas consideram fatores sociotécnicos na análise. Neste sentido, quando os fatores sociotécnicos são mencionados nas pesquisas, estes não são analisados em profundidade.

Uma forma de analisar os fatores sociotécnicos é por meio do framework proposto por Hendrick e Kleiner (2001) e ampliado por Guimarães (2009). Estes autores propuseram uma estrutura sociotécnica que abrange as diferentes perspectivas de Revista Produção Online, Florianópolis, SC, v.12, n. 4, p. 1106-1130, out./dez. 2012. 
análise necessárias. De acordo com esta abordagem, os sistemas sociotécnicos podem ser subdivididos em quatro subsistemas que estão inter-relacionados: (i) Subsistema Pessoal, que considera características das equipes, como profissionalismo, características demográficas e aspectos psicossociais; (ii) Subsistema Tecnológico, que considera o ambiente físico e as características do trabalho como equipamentos e sistemas de informações; (iii) Subsistema Organização do Trabalho, que envolve a forma em que foi projetado o trabalho, grau de centralização e formalização e as práticas utilizadas no trabalho, sendo projetado considerando as características dos dois subsistemas anteriores; e (iv) Subsistema do Ambiente Externo, no qual se consideram aspectos externos como: o ambiente socioeconômico, a educação, a política, características culturais e legais. Os três primeiros subsistemas descritos são influenciados este último subsistema.

Considerando as pesquisas anteriormente citadas sobre a utilização da TI conclui-se que uma análise mais aprofundada que considere essas quatro perspectivas sociotécnicas na utilização da TI é ainda necessária. Além disso, outro aspecto comum às pesquisas citadas acima é que elas destacam que quanto maior é a proximidade entre as equipes (co-localização) menor é a utilização da TI para a integração dos conhecimentos das mesmas, pois as equipes tendem a interagir mais face-a-face. No entanto, essas pesquisas não têm aprofundado nas diferenças apresentadas em termos de tipo de $\mathrm{Tl}$ e forma de utilização destas nas equipes virtuais e co-localizadas. Geralmente a análise concentra-se mais na intensidade de utilização da TI em ambos os tipos de equipes, considerando a TI como um conjunto de ferramentas tratadas de maneira genérica.

\section{MÉTODO DA PESQUISA}

Este trabalho focaliza-se em duas lacunas de pesquisa apontadas na revisão da literatura: (i) a falta de uma análise sociotécnica mais abrangente e (ii) a falta de um estudo que considere as diferenças da $\mathrm{Tl}$ em ambos os tipos de equipes de produto. A respeito do primeiro ponto, este trabalho utiliza a abordagem proposta por Hendrick e Kleiner (2001) e ampliada por Guimarães (2009) como estrutura de análise das Revista Produção Online, Florianópolis, SC, v.12, n. 4, p. 1106-1130, out./dez. 2012. 
características da utilização da TI. Por outro lado, a respeito do segundo ponto, este trabalho busca analisar as características de TI para a TC nos dois tipos de estratégias de equipes de projetos: equipes virtuais e equipes co-localizadas, realizando um comparativo entre as mesmas. Por tanto, para a realização da pesquisa assume-se como premissa que existem diferenças nas características da configuração de TI que se utiliza entre essas duas formas de organizar as equipes de projeto de produto. Isto significa que cada uma das estratégias de organização das equipes pode enfatizar diferentes tipos de $\mathrm{TI}$ assim como diferentes formas de combinar e utilizar as mesmas. Para verificar isto e identificar essas diferenças é desenvolvido um estudo de caso em duas empresas.

Considerando a natureza metodológica utilizada, a presente pesquisa classificase em aplicada, qualitativa e exploratória. De acordo com os procedimentos utilizados para a análise, foi utilizada a abordagem de estudo de caso (YIN, 2001). A seleção dos casos estudados foi por conveniência, sendo escolhidas duas empresas que operam no mesmo tipo de mercado, mas que possuem diferentes configurações das equipes de projeto. Uma destas empresas é composta principalmente por equipes virtuais e a outra principalmente por equipes co-localizadas. Ambas as empresas são multinacionais do setor de máquinas agrícolas e possuem centros de desenvolvimento de produto e fabricação no Brasil. Na Tabela 1 são apresentadas as características dos casos.

Tabela 1 - Características das empresas estudadas

\begin{tabular}{ccccc}
\hline $\begin{array}{c}\text { Casos } \\
\text { estudados }\end{array}$ & $\begin{array}{c}\text { Número de } \\
\text { funcionários } \\
\text { no Brasil }\end{array}$ & $\begin{array}{c}\text { Tamanho } \\
\text { da equipe } \\
\text { no Brasil }\end{array}$ & $\begin{array}{c}\text { Participantes da } \\
\text { pesquisa }\end{array}$ & $\begin{array}{c}\text { Principal forma de } \\
\text { organização }\end{array}$ \\
\hline Empresa A & 2000 & 60 & $\begin{array}{c}\text { - Coordenador de } \mathrm{TI}(1) \\
\text { - Engenheiros (3) }\end{array}$ & Equipes virtuais \\
\cline { 2 - 5 } Empresa B & 2500 & 100 & $\begin{array}{l}\text { - Gerente de projeto (1) } \\
\text { - Engenheiros (3) }\end{array}$ & $\begin{array}{l}\text { Equipes co- } \\
\text { localizadas }\end{array}$ \\
\hline
\end{tabular}

Como fontes de evidência do estudo utilizou-se uma triangulação dos dados com entrevistas, pesquisa documental e observação-participante, conforme a abordagem proposta por Yin (2001) para este tipo de estudos. Para as entrevistas foram escolhidas pessoas consideradas essenciais para o funcionamento dos projetos nas empresas estudadas. Os entrevistados pertencem a dois níveis hierárquicos da estrutura de Revista Produção Online, Florianópolis, SC, v.12, n. 4, p. 1106-1130, out./dez. 2012. 
gestão do desenvolvimento de produtos: o nível de coordenação das equipes e o nível de execução dos projetos. As entrevistas foram conduzidas de forma individual. Cada entrevista foi de aproximadamente uma hora, sendo cada uma delas realizadas em visitas diferentes. As entrevistas foram gravadas e transcritas para a coleta e análise dos dados. Para a condução das entrevistas utilizou-se um roteiro de entrevistas semiestruturadas (Apêndice A) e a estrutura de classificação da análise apresentada no Quadro 2 como suporte à discussão dos pontos de análise.

O Quadro 2 considera uma estrutura de análise da utilização da TI baseada na abordagem sociotécnica de Hendrick e Kleiner (2001) e Guimarães (2009). Além disso, este quadro considera diversas características da utilização da TI distribuídas em quatro subsistemas sociotécnicos. Essas características apresentadas no Quadro 2 baseiamse em aspectos observados por trabalhos anteriores conduzidos por Bartezcak et al. (2008), Corso et al. (2003), Corso e Paolucci (2001), Liao (2003) e Smith e Blanck (2002). Também, o trabalho apoiou-se em uma pesquisa realizada por Corso et al. (1999) que analisa a TI e TC em um contexto industrial similar ao considerado neste estudo.

Quadro 2 - Características para a observação no estudo de caso

\begin{tabular}{cl}
\hline \multicolumn{1}{c}{ Subsistemas } & \multicolumn{1}{c}{ Características de análise } \\
\hline \multirow{2}{*}{ Tecnologias } & - Tipo de TI utilizadas para a integração \\
& - Forma de utilização da TI para a integração e TC entre equipes \\
& - Complexidade, dificuldades e desafios tecnológicos \\
\hline \multirow{2}{*}{ Pessoas } & - Perfil dos Usuários \\
& - Formas de comunicação e integração com a TI utilizada \\
& - Dificuldades dos usuários em relação à TC por meio da TI \\
\hline \multirow{2}{*}{ Organização do trabalho } & - Etapas do projeto nas quais é utilizada a TI \\
& - Impacto da TI na organização das equipes \\
\hline Ambiente Externo & - Influência de fatores externos na utilização da TI nas equipes \\
\hline
\end{tabular}

Cada entrevista foi também acompanhada de uma visita ao ambiente de trabalho (observação-participante). Essas visitas tiveram uma duração de aproximadamente duas horas cada uma. Durante a observação-participante foi realizado também uma pesquisa documental dos registros de projetos, buscando assim entender como é o processo de trabalho das equipes. Também, acessou-se aos sistemas de informações 
utilizados pelas empresas para estudar a interface, forma de organização das informações e forma de utilização dos mesmos por parte das equipes. Durante estas visitas teve-se também contato com outros membros das equipes dos projetos com os quais se fizeram consultas sobre a forma de utilização dos sistemas de informações e ferramentas de TI disponíveis.

\section{RESULTADOS}

Nesta seção apresentam-se os resultados das duas empresas analisadas. Primeiro, nas subseções 5.1 e 5.2 apresentam-se as características de cada caso. Posteriormente, na subseção 5.3 realiza-se um comparativo entre as descrições dos dois casos.

\subsection{Análise da Empresa A (equipes virtuais)}

A Empresa A possui uma equipe de 60 pessoas vinculadas diretamente aos projetos de produto. Além disso, participam nos projetos outras pessoas de forma indireta, por meio de serviços terceirizados ou com parceiras. A principal estratégia de organização do trabalho é em equipes virtuais.

As equipes virtuais estão constituídas por participantes dispersos por diferentes regiões como Estados Unidos, Europa, Índia e Brasil. A maior parte dos produtos são desenvolvidos para uma estratégia global, sendo posteriormente adaptado às necessidades específicas de cada região. A empresa adotou a estratégia global para ganhar em flexibilidade de adaptação aos mercados e para aproveitar as características do conhecimento que possuem as filiais de cada região. Assim sendo, a parte da equipe que trabalha nos Estados Unidos se encarrega do planejamento estratégico e financeiro dos projetos. Os cálculos de engenharia são realizados na Índia. Na Europa trabalha-se com o projeto ergonômico e na América Latina realiza-se parte do projeto de engenharia, fabricação de motores, adaptações e montagem.

A empresa realiza importantes investimentos na área de TI para o trabalho com equipes virtuais, possuindo uma equipe específica para este fim. As características da Revista Produção Online, Florianópolis, SC, v.12, n. 4, p. 1106-1130, out./dez. 2012. 
TI utilizada para estas equipes é descrita a seguir, conforme a estrutura dos quatro subsistemas sociotécnicos apresentada no Quadro 2.

\subsubsection{Tecnologias utilizadas para a integração das equipes}

A empresa utiliza dois principais tipos de tecnologias para a integração das equipes: sistemas para troca de informações e sistemas de comunicação em tempo real. Para integrar as informações e fontes explícitas de conhecimentos entre os membros dos projetos utiliza-se como principal meio o sistema ERP (Enterprise Resource Planning) da empresa. Atualmente, a empresa está realizando um importante esforço para migrar todos os sistemas ao ERP. Com isto busca-se que o ERP integre todas as principais funções de processamento e armazenamento de informações da empresa, dentre elas as informações dos projetos de produto. Por meio deste sistema integrado, as diferentes equipes têm um acesso centralizado às fontes explícitas de conhecimento (por ex.: relatórios dos projetos, minutas de reuniões, registro de lições aprendidas, entre outros). Também, as informações e dados levantados nas diferentes fases do projeto são carregados a este sistema integrado, de maneira que as outras equipes possam acessar a estas informações de projeto.

Para o desenvolvimento dos produtos, as informações das equipes são carregadas ao sistema que centraliza estas informações em uma base de projetos virtuais. Os componentes do projeto são integrados virtualmente e todos os integrantes das equipes têm acesso remoto ao projeto completo. $O$ trabalho com protótipos virtuais baseados em sistemas CAD/CAE (Computer-Aided Design/Camputer-Aided Engineering) é fortemente utilizado. Por meio destes, as equipes distantes conseguem avaliar conjuntamente o funcionamento do produto antes da sua fabricação.

A empresa também utiliza portais corporativos e base de vídeos e fotografias em sistemas web para compartilhar novidades e conhecimentos entre os integrantes. Também são utilizados sistemas informativos digitais enviados por e-mail para disseminar os conhecimentos e resultados dos projetos para todos os participantes dos projetos. Além disso, as equipes utilizam diferentes tecnologias da comunicação destacando-se principalmente a vídeo-conferência, a teleconferência, os chats e eRevista Produção Online, Florianópolis, SC, v.12, n. 4, p. 1106-1130, out./dez. 2012. 
mails. Mediante estas tecnologias os integrantes realizam reuniões virtuais com os participantes de outros locais.

O principal desafio tecnológico com que a empresa lida atualmente é a integração de outros sistemas utilizados nos projetos dentro do ERP. A empresa tem realizado esforços para a implantação do ERP em todas as áreas e a nova meta é integrar todos os sistemas. Com isto seria possível realizar o trabalho de maneira totalmente remota, pois atualmente alguns sistemas devem ser utilizados apenas no ambiente de trabalho de cada centro de desenvolvimento. Além disto, com a integração do ERP o cruzamento de informações e disponibilidade de soluções similares de outros projetos poderia ter um melhor desempenho do que o atual.

\subsubsection{Pessoas envolvidas nos sistemas}

A utilização de TI para a integração das equipes é uma política estratégica da empresa para manter flexibilidade das equipes de trabalho independentemente do lugar onde estas se encontrem. Assim sendo, os integrantes estão acostumados a lidar com os aspectos tecnológicos da empresa. Além disso, a empresa possui uma equipe de $\mathrm{TI}$ que fornece todo o apoio necessário para os engenheiros de produto.

A maior discussão dos usuários da TI é acerca do nível de acesso às informações que cada integrante têm. Em alguns casos os participantes precisam de autorizações especiais para o acesso a informações necessárias para o trabalho dos projetos. Isto faz com que se prejudique a dinâmica de trabalho das equipes virtuais.

\subsubsection{Organização do trabalho}

A utilização da TI tem um forte impacto na organização do trabalho das equipes virtuais de projetos. O principal meio de TC e integração entre equipes é por meio destas ferramentas e toda a estrutura da gestão de projetos é baseada na utilização das mesmas. Contudo, as equipes também realizam uma parte do trabalho por meio de co-localização, pelo que o trabalho não é realizado completamente de maneira virtual. 


\subsubsection{Ambiente externo}

Em relação ao ambiente externo, as mudanças tecnológicas têm contribuído para o funcionamento das equipes virtuais. Principalmente 0 crescimento das tecnologias web que faz com que as equipes utilizem atualmente todos os softwares de gestão em servidores web com acesso remoto. Desta maneira alcançou-se uma redução significativa dos custos por licenças para usuários e facilitou-se o trabalho desde o campo para os engenheiros que se dedicam aos testes do produto in loco.

\subsection{Análise da Empresa B (equipes co-localizadas)}

A Empresa B possui uma equipe de aproximadamente 100 pessoas vinculadas diretamente aos projetos de produto. A principal estratégia de organização das equipes desta empresa é de co-localização. Do total de pessoas envolvidas no desenvolvimento dos projetos, aproximadamente a metade pertence à engenharia e trabalha em um único setor, compartilhando o mesmo ambiente de trabalho. O restante das pessoas pertence a outras áreas da mesma unidade da empresa. Cada unidade da empresa atende a produtos de uma determinada região.

$\mathrm{Na}$ empresa existe comunicação entre unidades de diferentes regiões, mas a prioridade do trabalho nos projetos é para as equipes co-localizadas. A empresa adotou esta estratégia para obter ganhos em conhecimento localizado, assim como no desenvolvimento de produtos mais focalizados para mercados específicos. Mesmo que a empresa trabalhe com equipes co-localizadas, foram realizados investimentos em diversos tipos de TI para o trabalho e TC das equipes. As características da TI para estas equipes é descrita a seguir, conforme a estrutura dos quatro subsistemas sociotécnicos apresentada no Quadro 2.

\subsubsection{Tecnologias utilizadas para a integração das equipes}

Nos últimos anos a empresa começou a realizar investimentos em $\mathrm{TI}$ como estratégia para a melhoria da comunicação e do controle interno das informações. Revista Produção Online, Florianópolis, SC, v.12, n. 4, p. 1106-1130, out./dez. 2012. 
Dentre as principais $\mathrm{TI}$ implantadas podem-se destacar: (i) sistema para o gerenciamento das informações dos projetos de produto; (ii) sistema para o gerenciamento das informações dos problemas de campo; e (iii) sistemas para comunicações entre equipes (notificações gerais, conferencias virtuais, etc.).

A principal preocupação da empresa em relação a sistemas de informações é disponibilizar as informações e fontes explícitas de conhecimentos para os engenheiros. Isto é realizado por meio de uma base de dados integrada em um sistema gerenciador. Este sistema se encarrega de gerenciar as atividades dos projetos em base a um modelo de desenvolvimento de produtos preestabelecido para todas as filiais da empresa. As equipes utilizam também ferramentas CAD/CAE, bases de dados e repositórios com fotos, vídeos e relatórios sobre os produtos, armazenados na intranet da empresa.

Uma das principais diferenças observadas em relação à Empresa $A$ é o método utilizado para a busca de conhecimentos nesta empresa. A forma de busca dessas fontes de conhecimento de outros projetos é por meio de conversas pessoais entre os integrantes. Assim sendo, os canais de acesso às informações por meio da TI são informais, utilizando-se a ajuda dos colegas para localizar as informações necessárias.

No entanto, com as ferramentas de TI abriram-se novos canais de comunicação que melhoraram a capacidade das pessoas manterem-se informadas sobre as ações globais. Os entrevistados destacaram que com isto as equipes conseguem saber o que está acontecendo em outros projetos, podendo identificar potenciais fontes de conhecimento que ajudariam a melhorar o desempenho do projeto. Além disso, um entrevistado explicou que, graças à implantação desses sistemas, os membros das equipes estão cientes dos resultados dos projetos antes das reuniões de equipes serem realizadas. Por conseguinte, no momento das reuniões eles são mais capazes de discutir sobre os problemas, tendo um melhor embasamento. Isto melhora a capacidade de discussão e de TC entre as pessoas que participam das reuniões.

Outro aspecto positivo destacado nas entrevistas foi a possibilidade de concentração das informações em um único local. Embora isto ainda não tenha sido totalmente solucionado, iniciou-se um processo de unificação das informações. Com isto foi melhorada a capacidade de identificação de potenciais fontes de conhecimento Revista Produção Online, Florianópolis, SC, v.12, n. 4, p. 1106-1130, out./dez. 2012. 
explícito para serem utilizadas nos projetos, pois os registros são identificados de maneira mais rápida. A empresa utiliza também sistemas ERP e outros sistemas para gerenciamento das informações dos processos.

Uma diferença importante entre a Empresa $A$ e $B$, é que esta última prioriza a construção de protótipos reais do produto, mesmo quando o projeto não está ainda suficientemente maduro. Com estes protótipos os engenheiros trabalham em grupos e aprendendo sobre o produto já montado, embora seja na sua versão parcial. Isto difere do que acontece na Empresa $A$, na qual a prioridade das equipes virtuais é dada ao trabalho em protótipos virtuais até o projeto estar bem avançado. Por outro lado, em relação às tecnologias da comunicação, na Empresa $B$ são utilizadas tecnologias similares às destacadas no caso da Empresa $A$, mas com um grau menor de intensidade. Em contraste, são priorizadas as reuniões presenciais e existe uma infraestrutura bem preparada para este tipo de atividades.

\subsubsection{Pessoas envolvidas nos sistemas}

A TC entre as equipes de projeto por meio do relacionamento pessoal é predominante no caso da Empresa B. A maior parte desse processo acontece de maneira desestruturada, por meio de conversações face-a-face, embora também sejam utilizadas algumas ferramentas de $\mathrm{TI}$ para este fim. Na implantação do novo sistema para gerenciamento das informações dos projetos ainda são necessárias melhorias da interface, de maneira que se facilite a utilização do mesmo pela equipe.

Observa-se que o sistema contém uma vasta gama informações, sendo difícil a visualização rápida das mesmas. Além disso, a utilização do sistema demanda tarefas específicas para carregar as informações no mesmo, o que demanda tempo das pessoas envolvidas nos projetos. Por este motivo, os entrevistados destacaram a necessidade futura de um funcionário dedicado especificamente ao gerenciamento deste sistema e ao controle das informações dos projetos. Por outro lado, atualmente estão sendo realizados treinamentos com os engenheiros sobre a utilização desses sistemas. Com isto busca-se criar uma cultura de TI ainda não bem estabelecida nesta empresa.

Revista Produção Online, Florianópolis, SC, v.12, n. 4, p. 1106-1130, out./dez. 2012. 


\subsubsection{Organização do trabalho}

Em relação à influência da TI na organização do trabalho, observa-se que a TI é utilizada principalmente como uma ferramenta de auxílio e não como um suporte que influencia na forma de organizar as atividades, como contraste ao caso da Empresa $A$. A utilização da TI como meio para TC e integração das equipes co-localizadas é usada principalmente nas atividades de retenção e armazenamento de fontes explícitas de conhecimento. Mesmo assim, pode-se observar que o ponto de maior impacto da $\mathrm{TI}$ sobre a organização é no controle dos processos por meio do sistema de gestão dos projetos utilizado.

Com este sistema, o trabalho das pessoas e as responsabilidades das atividades são controlados. Isto faz com que fique mais claro o papel de cada integrante e a forma com que cada um pode aportar para o restante das equipes, pois os demais integrantes sabem qual foi a tarefa atribuída para cada membro e, portanto, quais as experiências que estes tiveram que serviriam para contribuir aos novos projetos. Em síntese, a TI ajuda a esta empresa principalmente na coordenação das atividades do gerenciamento de multi-projetos.

\subsubsection{Ambiente externo}

As outras áreas desta empresa (por ex.: fornecedores, manufatura e marketing) têm influenciado sobre as decisões de implantação de novos sistemas de gerenciamento das informações dos projetos de produto. Isto se deve ao objetivo da empresa de ter um controle global das atividades das diferentes áreas em todas as unidades distribuídas em diferentes países.

Portanto, o ambiente externo aos projetos tem pressionado para implantar sistemas que controlem as atividades e que exijam realizar registros de lições aprendidas e de relatórios em um repositório de conhecimentos. Conforme destacaram os entrevistados, isto tornou às atividades do desenvolvimento de produto menos dinâmica, mas trouxe um grande avanço em termos de integração das informações e acesso às entre as diferentes áreas da empresa.

Revista Produção Online, Florianópolis, SC, v.12, n. 4, p. 1106-1130, out./dez. 2012. 


\subsection{Comparações das características de TI}

Em base à descrição dos dois casos analisados anteriormente, no Quadro 3 destacam-se as principais diferenças e semelhanças observadas na utilização da TI nas equipes virtuais e co-localizadas. Nessas características observam-se aspectos tais como o nível maior de investimento em TI por parte das equipes virtuais, o foco de utilização de TI de cada um dos tipos de equipes e as contribuições do meio ambiente externo para o desenvolvimento das equipes virtuais. 
Quadro 3 - Comparação de resultados dos dois casos estudados

\begin{tabular}{|c|c|c|c|}
\hline $\begin{array}{c}\text { Sub- } \\
\text { sistemas }\end{array}$ & $\begin{array}{l}\text { Elementos } \\
\text { analisados }\end{array}$ & Empresa A (equipes virtuais) & Empresa B (equipes co-localizadas) \\
\hline \multirow{5}{*}{ 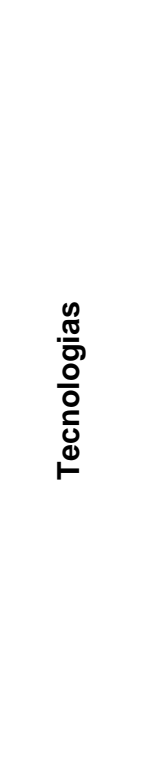 } & $\begin{array}{l}\text { Foco principal } \\
\text { da TI para TC }\end{array}$ & $\begin{array}{l}\text { Integração e comunicação de equipes } \\
\text { e integração de sistemas no ERP. }\end{array}$ & $\begin{array}{l}\text { Armazenamento de conhecimentos } \\
\text { explícitos e coordenação de atividades. }\end{array}$ \\
\hline & $\begin{array}{l}\text { Principal tipo de } \\
\text { TI utilizado }\end{array}$ & $\begin{array}{l}\text { Sistemas ERP e outros sistemas web. } \\
\text { Integram as informações das equipes } \\
\text { remotas. Decisões e controle } \\
\text { centralizados em um único sistema. } \\
\text { Também há uma utilização intensiva } \\
\text { de ferramentas de comunicação em } \\
\text { tempo real. }\end{array}$ & $\begin{array}{l}\text { Repositórios de conhecimentos e } \\
\text { sistemas para coordenação dos } \\
\text { projetos. Porém, as bases de dados } \\
\text { estão localizadas em cada grupo de } \\
\text { trabalho o que está sendo mudado nos } \\
\text { últimos anos. }\end{array}$ \\
\hline & $\begin{array}{l}\text { Nível de } \\
\text { investimento em } \\
\text { TI }\end{array}$ & $\begin{array}{l}\text { Alto. Principalmente investimentos em } \\
\text { projetos virtuais e ferramentas de } \\
\text { simulação que permitem trabalhar com } \\
\text { equipes distantes. }\end{array}$ & $\begin{array}{l}\text { Médio. Principalmente em } \\
\text { transformação das documentações para } \\
\text { meios eletrônicos de fácil identificação e } \\
\text { acesso. }\end{array}$ \\
\hline & $\begin{array}{l}\text { Experiência em } \\
\text { utilização de TI }\end{array}$ & $\begin{array}{l}\text { Alta. A empresa possui uma tradição e } \\
\text { uma equipe de TI para os } \\
\text { investimentos em esta área. }\end{array}$ & $\begin{array}{l}\text { Média. Não há uma forte experiência em } \\
\text { esta área. Muitos dos serviços são } \\
\text { terceirizados. }\end{array}$ \\
\hline & $\begin{array}{l}\text { Complexidade, } \\
\text { dificuldades e } \\
\text { desafios } \\
\text { tecnológicos }\end{array}$ & $\begin{array}{l}\text { Algumas limitações para integrar } \\
\text { sistemas com o ERP da empresa, } \\
\text { sendo necessária a utilização de } \\
\text { interfaces. }\end{array}$ & $\begin{array}{l}\text { Algumas limitações na implantação do } \\
\text { sistema gerenciador dos projetos. } \\
\text { Dificuldade cultural e necessidade de } \\
\text { treinamentos intensivos na área de TI. }\end{array}$ \\
\hline \multirow{3}{*}{ 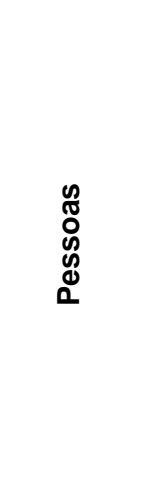 } & $\begin{array}{l}\text { Perfil dos } \\
\text { Usuários }\end{array}$ & $\begin{array}{l}\text { Os usuários estão acostumados com a } \\
\text { integração por meio de tecnologias } \\
\text { visto que isto faz parte de uma } \\
\text { estratégia adotada há vários anos. }\end{array}$ & $\begin{array}{l}\text { A utilização de TI gerencial (não } \\
\text { operacional como sistemas CAD/CAE) é } \\
\text { nova no grupo. Portanto, ainda está em } \\
\text { um processo de adaptação. }\end{array}$ \\
\hline & $\begin{array}{l}\text { Formas de } \\
\text { comunicação e } \\
\text { integração com } \\
\text { a TI utilizada } \\
\end{array}$ & $\begin{array}{l}\text { Comunicação intensa por meio de } \\
\text { ferramentas de comunicação em } \\
\text { tempo real (por ex.: videoconferências } \\
\text { e chats). }\end{array}$ & $\begin{array}{l}\text { Utilização de arquivos das bases de } \\
\text { dados de outros grupos de trabalho. }\end{array}$ \\
\hline & $\begin{array}{l}\text { Dificuldades dos } \\
\text { usuários em } \\
\text { relação à TC } \\
\text { por meio da TI }\end{array}$ & $\begin{array}{l}\text { Perda de confiança pela falta de } \\
\text { interação direta face-a-face. }\end{array}$ & $\begin{array}{l}\text { Os usuários têm dificuldade de } \\
\text { identificar fontes explícitas de } \\
\text { conhecimento, pois as bases não estão } \\
\text { totalmente integradas. }\end{array}$ \\
\hline 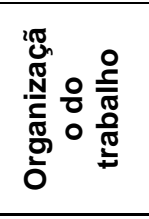 & $\begin{array}{l}\text { Contribuição da } \\
\text { TI para a TC } \\
\text { entre as } \\
\text { equipes }\end{array}$ & $\begin{array}{l}\text { Permite um acesso global às fontes de } \\
\text { conhecimento explícito dos projetos. } \\
\text { Contudo, a empresa organiza as } \\
\text { equipes virtuais principalmente por } \\
\text { macro-etapas do projeto para não } \\
\text { perder contato face-a-face. }\end{array}$ & $\begin{array}{l}\text { A TI ajuda na coordenação do trabalho } \\
\text { das equipes, facilitando a sincronização } \\
\text { das atividades multi-projetos. }\end{array}$ \\
\hline 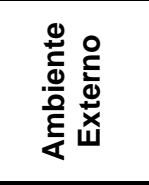 & $\begin{array}{l}\text { Influência de } \\
\text { fatores externos } \\
\text { na utilização da } \\
\text { TI }\end{array}$ & $\begin{array}{l}\text { O crescimento das opções web facilita } \\
\text { a integração das equipes virtuais. }\end{array}$ & $\begin{array}{l}\text { O controle corporativo, com matriz nos } \\
\text { EUA, exige a implantação de TI para o } \\
\text { seguimento das etapas dos projetos e } \\
\text { das informações compartilhadas nas } \\
\text { equipes. }\end{array}$ \\
\hline
\end{tabular}

No Quadro 3 observa-se que a Empresa B (equipes co-localizadas) não tem um grau de desenvolvimento e utilização de TI tão elevado quanto no caso da Empresa $A$ (equipes virtuais). No entanto, a Empresa $B$ está realizando um importante esforço Revista Produção Online, Florianópolis, SC, v.12, n. 4, p. 1106-1130, out./dez. 2012. 
nesta direção, buscando se tornar mais competitiva por meio da sua infraestrutura de TI. Neste caso a motivação para o investimento em TI é diferente da Empresa A. A motivação da Empresa B para investir em TI é buscar desenvolver a capacidade de reter conhecimentos ao longo do tempo. Embora as pessoas trabalhem no mesmo ambiente de trabalho e tenham a facilidade de discutir face-a-face e compartilhar conhecimentos rapidamente entre os participantes, a forma com que isto é realizado é informal. Portanto, uma importante parte deste conhecimento é perdida ao longo do tempo.

Por outro lado, observou-se na Empresa A (equipes virtuais) que o fato das equipes trabalharem distanciadas faz com que normalmente sejam utilizadas mais fontes de conhecimento explícito, muitas vezes por meio de sistemas de gerenciamento de informações ou simplesmente por meio de e-mails que são enviados aos participantes. Contudo, o interesse deste tipo de equipes não se centra apenas no armazenamento dos conhecimentos, mas nos meios de comunicação para aproximar as pessoas e superar as barreiras de interação naturais neste tipo de equipes.

Com estas comparações descritas observa-se que ambas as estruturas de equipes, virtuais (Empresa A) e co-localizadas (Empresa B), têm um importante interesse na $\mathrm{TI}$, embora tenham objetivos de utilização diferentes. Desta maneira, a estrutura comparativa apresentada pode ajudar outras empresas que desejam desenvolver uma estratégia de utilização de TI para a TC. Isto é devido ao fato que a mesma permite identificar elementos importantes a serem considerados em cada uma das estruturas de equipes de projetos adotadas.

\section{CONSIDERAÇÕES FINAIS}

Por meio de uma análise de dois casos de empresas multinacionais, foram analisadas as características de TI para a TC em dois tipos de estratégias de equipes de projetos (equipes virtuais e equipes co-localizadas) e foi realizado um comparativo entre as mesmas, destacando a forma de utilização de TI em cada estratégia. A análise baseou-se na abordagem sociotécnica que considera quatro subsistemas interrelacionados: tecnologias, pessoas, organização do trabalho e ambiente externo. Esses Revista Produção Online, Florianópolis, SC, v.12, n. 4, p. 1106-1130, out./dez. 2012. 
quatro subsistemas permitiram analisar os casos desde diferentes perspectivas de utilização da TI.

Os resultados obtidos mostraram diferenças nas abordagens utilizadas para a utilização da $\mathrm{TI}$ das equipes virtuais e co-localizadas. Observou-se que as equipes virtuais priorizaram a TI como meio para superar as distâncias físicas e culturais entre os participantes de diferentes países. Já, as equipes co-localizadas priorizaram a utilização da TI como meio para a retenção de conhecimento em fontes explícitas, visto que as equipes trabalham juntas e, portanto, a TC acontece principalmente por canais informais que levam ao risco de perda do conhecimento.

Contribuições

A principal contribuição acadêmica dos resultados apresentados é a ampliação do entendimento da realidade das equipes virtuais e co-localizadas a respeito da forma em que estas utilizam as diferentes ferramentas de TI. A maior parte dos estudos disponíveis na literatura têm realizado pesquisas de levantamento (survey) que não aprofundam sobre as características particulares de cada tipo de estratégia de trabalho em equipes. Além disso, geralmente esses estudos destacam a importância da TI, mas não descrevem com as mesmas são utilizadas para a TC dentro dessas estratégias de equipes. Portanto, os resultados apresentados permitem ampliar o entendimento sobre a contribuição da TI para a TC desde uma perspectiva prática e mais aprofundada na realidade das empresas.

Como contribuição prática, este trabalho permite que os gerentes de $\mathrm{Tl}$ e de desenvolvimento de produto possam ter uma noção mais clara das diferentes abordagens existentes para a aplicação da TI como suporte à TC entre as equipes de produto. Isto pode ajudar à seleção das ferramentas de TI mais apropriadas para uma determinada estrutura de equipe de produto. Também, pode ajudar a alinhar a estrutura das equipes de projetos com o tipo de TI disponível na empresa. Além disso, os resultados apresentados mostram diferentes elementos que os gerentes deveriam considerar quando desejam desenvolver uma estratégia de trabalho das equipes focalizada na TC e suportada pela estrutura de TI.

Limitações e Pesquisas Futuras

Revista Produção Online, Florianópolis, SC, v.12, n. 4, p. 1106-1130, out./dez. 2012. 
Uma limitação do trabalho é que foram abordados apenas dois casos práticos. No entanto, o objetivo do presente trabalho foi precisamente aprofundar em poucos casos para obter um maior grau de detalhamento de informações que um estudo tipo survey não permite alcançar. A partir disto, futuras pesquisas podem ampliar o escopo desta pesquisa, buscando analisar este tema em outros contextos industriais e/ou com um número maior de casos. Outra opção é também estender os resultados aqui apresentados por meio de uma pesquisa mais abrangente, do tipo survey, e testar as possíveis diferenças com os resultados aqui apresentados. Desta maneira, o presente trabalho serve com norteador para que futuras pesquisas ampliem o entendimento sobre esta questão.

\section{AGRADECIMENTOS}

Os autores agradecem ao CNPq e FAPERGS pelo apoio financeiro recebido para o desenvolvimento desta pesquisa.

\section{REFERÊNCIAS}

AKGÜN, A.E.; DAYAN, M.; BENEDETTO, A.di. New product development team intelligence: antecedents and consequences. Information \& Management, v.45, p.221226, 2008.

ALAVI, M.; LEIDNER, D.E. Knowledge management and knowledge management systems: conceptual foundations and research issues. MIS Quarterly Review, v.25, n.1, p.107-136, 2001.

AOSHIMA, Y. Transfer of system knowledge across generations in new product development: empirical observations from japanese automobile development. Industrial Relations, v.41, n.4, p.605-628, 2002.

BARCZAK, G.; HULTINK, E. J.; SULTAN, F. Antecedents and consequences of information technology usage in npd: a comparison of dutch and u.s. companies. Journal of Product Innovation Management, v. 25, p.620-631, 2008.

BARTEZZAGHI, E.; CORSO, M.; VERGANI, R. Continuous improvement and interproject learning in new product development. International Journal of Technology Management, v.14, n.1, p.116-138, 1997. 
BASKERVILLE, R.; DULIPOVICI, A. The theoretical foundations of knowledge management. Knowledge Management Research \& Practice, v.4, p.83-105, 2006.

BOER, H.; CAFFYN, S.; CORSO, M.; COUGHLAN, P; GIESKES, J.; MAGNUSSON, M.; PAVESI, S.; RONCHI, S. Knowledge and continuous innovation: the CIMA methodology. International Journal of Operations \& Production Management, v.21, n.4, p.490-503, 2001.

CHOI, B.; LEE, H. An empirical investigation of KM styles and their effect on corporate performance. Information and management, v.40, p.403-417, 2003.

CORSO, M.; MARTINI, A.; PELLEGRINI, L.; PAOLUCCI, E. Technological and organizational tools for knowledge management: in search of configurations. Small Business Economics, v.21, n.4, p.397-408, 2003.

CORSO, M.; MUFFATTO, M.; VERGANTI, R. Reusability and multi-product development policies: a comparison of approaches in the automotive, motorcycle and earthmoving machinery industries. Robotics and Computer-Integrated Manufacturing, v.15, n. 1, p. 155-165, 1999.

CORSO, M.; PAOLUCCI, E. Fostering innovation and knowledge transfer in product development through information technology. International Journal of Technology Management, v.22, n. 1-3, p. 126-148, 2001.

CORSO, M.; PAVESI, S. How management can foster continuous product innovation. Integrated Manufacturing Systems, v.11, n.3, p.199-211, 2000.

CUSUMANO, M.A.; NOBEOKA, K. Thinking beyond Lean. MIT Motor Vehicle Program, Ney York: The Free Press, 1998.

CUMMINGS, J.L.; TENG, B.S. Transferring R\&D knowledge: the key factor affecting knowledge transfer success. Journal of Engineering and Technology Management, v.20, n.1-2, p.39-68, 2003.

DAVENPORT, T. H.; VÖLPEL, S.C. The rise of knowledge towards attention management. Journal of Knowledge Management, v.5, n.3, p.212-221, 2001.

DAVENPORT, T.H.; PRUSAK, L. Working knowledge. Boston: Harvard Business Scholl Press, 1998. 199p.

FARSHCHIAN, B. A. Integrating geographically distributed development teams through increased product awareness. Information Systems, v. 26, n. 7041, p. 123-141, 2001. 
GUIMARÃES, L. B. de M. The practice of ergonomics in the south of Brazil from a sociotechnical perspective. In: SCOTT, Pat. (Org.). Ergonomics in developing countries. London: Taylor and Grancis, v. 1, 2009.

HENDRICK, H.W; KLEINER, B.M. Macroergonomics: an introduction to work system design. Santa Monica, CA: Human Factors and Ergonomics Society, 2001. 417p.

JOSHI, K. D.; SARKER, S.; SARKER, S. Knowledge transfer within information systems development teams: examining the role of knowledge source attributes. Decision Support Systems, v. 43, p. 322 - 335, 2007.

LAKEMOND, N.; BERGGREN, C. Co-locating NPD? The need for combining project focus and organizational integration. Technovation, v. 26, n. 7, p. 807-819, 2006.

LIAO, S-H. Knowledge management technologies and applications - literature review from 1995 to 2002. Expert Systems with Applications, v.25, P.155-164, 2003.

LIN, H-F; LEE, G-G. Effects of socio-technical factors on organizational intention to encourage knowledge sharing. Management Decision, v. 44, n.1, p.74-88, 2006.

LIU, D-R.; KE, C-K. Knowledge support for problem-solving in a production process: a hybrid of knowledge discovery and case-based reasoning. Expert systems with applications, v.33, n.1, p.147-161, 2007.

LYNN, G.S.; REILLY, R.R.; AKGÜN, A.E. Knowledge management in new product teams: practices and outcomes. IEEE Transactions on Engineering Management, v.47, n.2, p.221-231, 2000.

MARSH, S. J.; STOCK, G. N. Creating dynamic capability: the role of intertemporal integration, knowledge retention, and interpretation. Journal of Product Innovation Management, p. 422-436, 2006

MONTOYA, M. M.; MASSEY, A. P.; HUNG, Y. C.; CRISP, C. B. Can you hear me now? communication in virtual product development teams. Journal of Product Innovation Management, p. 139-155, 2009.

NOBEOKA, K.; CUSUMANO, M. A. Multiproject strategy and sales growth: the benefits of rapid design transfer in new product development. Strategic Management Journal, v. 18, n. 3, p. 169-86, 1997.

NONAKA, I. A. dynamic theory of organizational knowledge creation. Organization Science, v.5, n.1, p.14-37, 1994.

PRENCIPE, A.; TELL, F. Inter-project learning: processes and outcomes of knowledge codification in project-based firms. Research Policy, v.30, n.9, p.1373-1394, 2001. 
RAMESH, B.; TIWANA, A. Supporting collaborative process knowledge management in new product development teams. Decision Support Systems, 1999.

SARKER, Saonee; SARKER, Suprateek; NICHOLSON, D.B.; JOSHI, K. Knowledge transfer in virtual system development teams: an exploratory study of four key enablers. IEEE Transactions on Professional Communication, v.48, n.2, p.201-218, 2005.

SCARBROUGH, H.; SWAN, J.; LAURENT, S.; ET AL. Project-Based Learning and the Role of Learning Boundaries. Organization Studies, v. 25, p. 1579-1600, 2004.

SMITH, P.G.; BLANCK, E.L. From experience: leading dispersed teams. Journal of Product Innovation Management, v.19, p.294-304, 2002.

SÖDERQUIST, K.E. Organising knowledge management and dissemination in new product development. Long Range Planning, v.39, n. 5, p.497-523, 2006.

SONG, M.; BIJ, H. V.; WEGGEMAN, M. Determinants of the Level of Knowledge Application: A Knowledge-Based and Information-Processing Perspective. Journal of Product Innovation Management, v. 22, p. 430-444, 2005.

SONG, M.; BERENDS, H.; BIJ, H. V.; WEGGEMAN, M. The effect of it and co-location on knowledge dissemination. Journal of Product Innovation Management, v. 24, p. 52-68, 2007.

TIWANA, A.; RAMESH, B. A design knowledge management system to support collaborative information product evolution. Decision Support Systems, v.31, p.241262, 2001.

TSENG, S. The effects of information technology on knowledge management systems. Expert Systems with Applications, v. 35, n. 1-2, p. 150-160, 2008.

YIN, R. K. Estudo de caso: planejamento e métodos. Porto Alegre: Bookman, 2001.

ZHENGFENG, L.; JINFU, Y.; YAN, Z. An empirical study on the effect mechanism of knowledge management on new product development in aviation industry. In: INTERNATIONAL CONFERENCE ON WIRELESS COMMUNICATIONS, NETWORKING AND MOBILE COMPUTING (WiCom). Anais... . p.5460-5463. Shangai, China: IEEE, 2007.

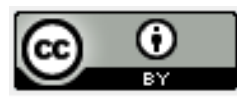

Artigo recebido em 23/05/2011 e aceito para publicação em 13/08/2012. 


\section{Apêndice A - Roteiro de Entrevista Semi-Estruturada}

\section{Primeira parte: Características Gerais}

Nome da pessoa:

Função na gestão dos projetos:

Tempo que atua dentro do departamento:

A- Características gerais da empresa:

A.1) Qual é o foco do Negócio da empresa?

A.2) Que tipos de produtos são desenvolvidos? Qual é a principal linha de produto da empresa?

A.3) Como é a estrutura de trabalho das equipes de projeto?

A.4) Como é organizada a gestão dos projetos de produto (definição de líderes, pessoas envolvidas, determinação de recursos e prazos de execução)?

A.5) Como são coordenadas as atividades dos diferentes projetos?

B-Segunda Parte: Utilização das informações e do conhecimento nos projetos de produto:

B.1) Que tipos de relatórios, documentos de lições aprendidas ou similares são gerados nos projetos?

B.2) Onde são armazenadas essas informações e documentos (bases de dados, sistemas informatizados, arquivos impressos, etc.)? Como é o acesso aos mesmos? Existem fontes de informação e conhecimento comuns a todos os projetos?

B.3) Como é a relação entre diferentes projetos? Existe integração? Como é dada essa integração?

B.4) Como são utilizadas as informações dos outros projetos e a documentação de projetos passados? Outros projetos utilizam informações registradas dos demais projetos?

B.5) Existe alguma fase específica dos projetos que se destaque por ser intensiva em utilização de informações de outros projetos ou de experiências e conhecimentos de pessoas envolvidas em outros projetos ou em projetos passados?

B.6) Existem ferramentas ou práticas utilizadas com a finalidade de compartilhar informações e conhecimento entre diferentes projetos?

B.7) Existem pessoas-chaves que ajudam a disseminar as informações e o conhecimento entre diferentes projetos? Existem pessoas que atuam como fonte de conhecimento para dar suporte a diferentes projetos?

C-Terceira Parte: Utilização de TI para a integração de conhecimentos entre as equipes

Esta terceira parte é norteada pelas características descritas na Figura 3. 